\title{
The impact of telemonitoring upon hospice referral in the community: a randomized controlled trial
}

\author{
Paul Y Takahashi' \\ Gregory J Hanson 1,2 \\ Bjorg Thorsteinsdottirl \\ Holly K Van Houten ${ }^{3}$ \\ Nilay D Shah ${ }^{3}$ \\ James M Naessens ${ }^{3}$ \\ Jennifer L Pecina ${ }^{4}$ \\ 'Division of Primary Care Internal \\ Medicine, ${ }^{2}$ Kogod Center of Aging, \\ ${ }^{3}$ Division of Health Science Research, \\ ${ }^{4}$ Department of Family Medicine, \\ Mayo Clinic, Rochester, MN, USA
}

\author{
This article was published in the following Dove Press journal: \\ Clinical Interventions in Aging \\ 30 October 2012 \\ Number of times this article has been viewed
}

Background: Using telemedicine for older adults with multiple comorbid conditions is a potential area for growth in health care. Given this older, ailing population, providers should discuss end-of-life care with patients.

Objective: To determine the relationship between telemonitoring and hospice enrollment compared to usual care among older adults with chronic health problems.

Methods: This was a secondary evaluation of a randomized controlled trial. The trial was performed at an academic medical center. Patients who were over the age of 60 and had a high risk of hospitalization and emergency department visits were recruited to the study. The primary outcome was hospice enrollment, and the secondary outcome was the mean number of days in hospice. The data were analyzed using Chi-squared tests and time-to-event analysis.

Results: The average age of the cohort was 80.3 years. Nine patients $(9.6 \%)$ in the telemonitoring group were enrolled in hospice care, whereas four patients $(4.0 \%)$ in the usual care group were enrolled $(P=0.12)$. The mean number of days in hospice was $57.9(\mathrm{SD} \pm 99.2)$ for the telemonitoring group, and 119.3 ( $\mathrm{SD} \pm 123.8)$ for the usual care group $(P=0.36)$. There was no significant difference regarding time to hospice referral.

Conclusion: In this pilot analysis, there were no differences noted between groups in the number of patients that entered into hospice or the amount of time they stayed in hospice care. This was a small trial, and the power to detect a difference was 36\%. It was encouraging that twice the number of patients enrolled in hospice care in the telemonitoring group compared to usual care despite the insignificant finding. Further research may determine the effect of telemonitoring upon hospice referral.

Keywords: hospice, elderly, telemonitoring, palliative care

\section{Introduction}

Case management and telemonitoring are important clinical practice strategies for older adults with chronic health conditions. Indeed, many patients have clinically advanced conditions that require intensive monitoring, and this may sometimes be completed through the use of telemonitoring. For older at-risk adults, providers will likely place greater emphasis on case management. In many previous telemonitoring trials, investigators studied hospitalization ${ }^{1,2}$ or process measures like hemoglobin $\mathrm{A}_{1 \mathrm{c}}$ levels. ${ }^{3}$ Patients care about these outcomes; however, researchers have not evaluated the effects of telemonitoring on hospice enrollment. Risk-stratified patients receiving telemonitoring suffer chronic medical conditions that place them at risk for hospitalization. These chronically ill patients may require hospice care. In this medically complex cohort, mortality may be as high as $22 \%$ over a 2 -year time period. ${ }^{4}$
Correspondence: Paul Y Takahashi Division of Primary Care Internal Medicine, Mayo Clinic, 200 First Street SW, Rochester, MN 55905, USA

Tel + I 50728425 I I

Fax + I 5072660036

Email takahashi.paul@mayo.edu 
Providers may encourage end-of-life discussions while patients are under intense disease monitoring, as both the provider and the patient learn more about the prognosis of the chronic illness.

One important aspect of telemonitoring is that it may be a potential route through which to offer hospice services to appropriate patients. The literature describes telemonitoring in hospice care after patients have been enrolled in hospice. It is evident that hospice visits through the use of a videophone may improve the quality of life for hospice patients. ${ }^{5}$ With the frequent monitoring of patients, one might expect that a patient's prognosis and the options surrounding hospice care (if appropriate) might be discussed. However, it is unclear whether intensive patient management using telemonitoring alone influences hospice referrals. To answer this question, we performed a secondary analysis of the data from a previous randomized controlled trial $^{6}$ to assess the impact of telemonitoring versus usual care in hospice admissions among older at-risk adults in the community. We hypothesized that older adults receiving telemonitoring will have a higher rate of hospice referrals compared to those receiving usual care.

\section{Methods}

The specific details of the study protocol have been published previously. ${ }^{7}$ The following methods provide an overview of the study. This study was approved by the Mayo Clinic Institutional Review Board.

\section{Design}

This was a randomized controlled trial of older adults accessing health care services at four sites in southeastern Minnesota. Three sites were in Rochester and one was in rural Kasson. Changes to the trial (enrollment, design, outcomes) were not made after initiation of the study.

\section{Participants}

All subjects were over the age of 60 and had a primary care provider at the Mayo Clinic. Subjects were stratified based on an Elder Risk Assessment (ERA) score above $15 .^{8}$ Participants were selected if they were considered to be at high risk for future hospital stays. Patients' ERA scores were based on age, days of previous hospitalization, and comorbid conditions like heart disease, diabetes, chronic obstructive pulmonary disease, and dementia.

\section{Exclusion}

Patients were excluded from the study if they were diagnosed with dementia during pre-enrollment screening.
They were also excluded if they were enrolled in a hospice prior to randomization; this information was obtained via self-report. Patients were excluded if they could not speak English. Patients unable to use the telemonitoring device were also excluded.

\section{Interventions}

The Intel ${ }^{\circledR}$ Health Guide (Intel Corporation, Santa Clara, CA) is a Food and Drug Administration-approved device that was used daily by the intervention group. Patients in the telemonitoring group relayed biometric and clinical information asynchronously to a registered nurse, who reviewed this information daily. When required, the medical care team would communicate with the patient via phone or videoconferencing. The telemonitoring program did not include a specific program, checklist, guideline, or algorithm for enrollment into hospice care. Patients were enrolled in hospice care when deemed clinically appropriate by the primary care doctor.

Usual care was applied to both the telemonitoring and usual care groups. Patients in both groups had full access to primary and specialty care. They had access to a nurse by telephone, they also had access to the emergency department, and they were provided with follow-up phone calls after a hospital visit. In addition, patients had access to in-hospital palliative care, outpatient palliative care, and hospice care.

\section{Outcomes}

While enrolled in the study, the primary outcome measured was hospice referral. Patients and their primary physician made the mutual clinical decision for hospice entry. Secondary outcomes included the amount of time spent in hospice, measured both as a mean and median, and the time to hospice referral after enrollment in the study. The referral to any hospice was clearly documented in the electronic medical record (EMR).

\section{Predictor variables}

At baseline, initial characteristics were compared between groups. These included demographic factors (age, sex) and functional factors (grip strength, gait speed, and results on the Timed Up and Go test). The predictors also included the scores from the following assessment measures: the ERA, ${ }^{8}$ the Kokmen Short Test of Mental Status (dementia), ${ }^{9}$ the SF-12 ${ }^{\circledR}$ (mental and physical subscales for quality of life; Mental Outcomes Trust, Hanover, NH), ${ }^{10}$ the Patient Health Questionnaire nine-item scale (depression), and the Barthel Index. ${ }^{11}$ We measured grip strength using a gripstrength dynamometer. ${ }^{12}$ Gait speed was reported in meters 
per second; gait speed of less than 1 meter per second has previously been associated with increased mortality. ${ }^{13}$ The Timed Up and Go test involves having a patient start from a seated position, stand, walk, turn, and return to his or her seat. The Timed Up and Go test has commonly been used as a predictor for falls. ${ }^{14,15}$

\section{Data collection}

The outcomes data were collected directly from each patient's clinical chart using the EMR. The Mayo Clinic has a comprehensive EMR that records all encounters with the patient. The entry of patients into hospices was clearly recorded.

\section{Sample size}

The sample size was designed to evaluate hospitalizations. ${ }^{6}$ This study represents a secondary analysis of this cohort, and was not initially powered to look directly at hospice enrollment.

\section{Randomization and blinding}

The study used block randomization based on each patient's site of primary care enrollment. The blocks encompassed two to four individuals. The method of randomization was computer-generated by a statistician who was not involved with the analysis. The randomization decisions were placed in sequential, sealed envelopes. This study was not blinded because of the practical limitations of the intervention. Analysis of the outcomes was blinded to group type.

\section{Statistical methods}

Descriptive statistics will be used to review the characteristics of the overall cohort. Our previous study revealed that there were no baseline differences between the telemonitoring group and the usual care group, and no adjustments to the outcomes were made. ${ }^{6}$ The demographics, comorbid conditions, and the patient's overall quality of life will be reported and compared between the intervention group and the usual care group at baseline. The primary outcome of hospice enrollment will be analyzed using logistic regression analysis. Time-to-event analysis will be used to examine the time to hospice referral and time to death in hospice patients. All tests will assume a two-sided $P$-value with significance at 0.05 . All analyses were run on SAS 9.2 (SAS Institute, Inc, Cary, NC).

\section{Results}

A total of 205 participants were enrolled in the study. The characteristics of this cohort have been previously discussed. ${ }^{6}$ The initial flow of patients is noted in Figure $1 .{ }^{6}$ The study

\begin{tabular}{l}
\hline 513 phone calls to high-risk older adults. \\
- 234 interested \\
- 217 not interested \\
- 16 problems with machine use \\
- 14 did not plan on staying in area \\
- 32 other causes for refusal \\
\hline
\end{tabular}

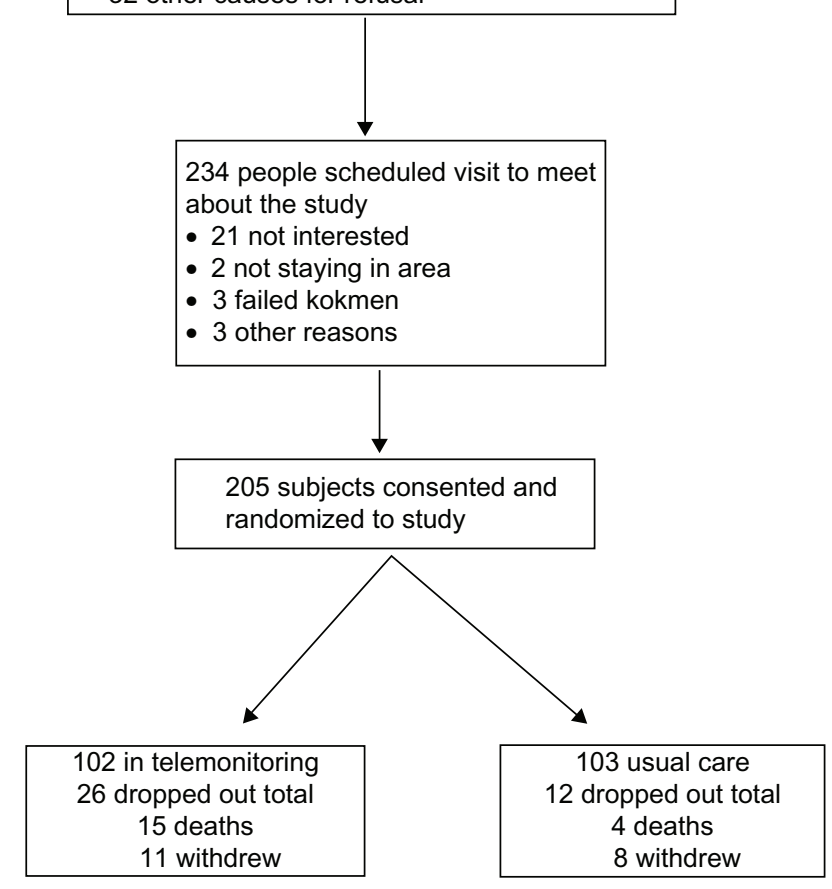

Figure I Flow of patients from recruitment to randomization for 205 patients over 60 years.

Note: Copyright (C) 2012, American Medical Association. All rights reserved. Adapted with permission from Takahashi PY, Pecina JL, Upatising B, et al. A randomized controlled trial of telemonitoring in older adults with multiple health issues to prevent hospitalizations and emergency department visits. Arch Intern Med. 20I2; I72(I0):773-779.

was conducted from November 2009 until July 2011 and was completed when both the enrollment and time goals were reached. There were no clinical differences between the intervention group and the usual care group (Table 1). The SF- $12^{\circledR}$ mental scores were slightly different between both groups; however, the results were not adjusted as a result of this difference.

Regarding the primary outcome of hospice referral, nine patients $(9.6 \%)$ in the telemonitoring group were enrolled in hospice care, compared to four patients $(4.0 \%)$ in the usual care group, although this result was insignificant $(P=0.12)$. The mean number of days from time of enrollment in the trial and concluding with entry into hospice care was not different between the two groups. The mean number of days in hospice care tended to be higher in the usual care group with an average of 119.3 days ( $\mathrm{SD} \pm 123.8$ days) when compared to the intervention group, who stayed in hospice for an average of 57.9 days ( $\mathrm{SD} \pm 99.2$ days). The median numbers of days in hospice care were 106.0 days and 27.0 days, respectively. These findings are noted in Table 2. 
Table I Baseline characteristics of overall group and by randomized group for 205 patients

\begin{tabular}{|c|c|c|c|c|}
\hline Characteristic & $\begin{array}{l}\text { Total } \\
n=205\end{array}$ & $\begin{array}{l}\text { Telemonitoring } \\
\mathrm{n}=102\end{array}$ & $\begin{array}{l}\text { Usual care } \\
\mathrm{n}=103\end{array}$ & $P$-value \\
\hline Age & $80.3 \pm 8.2$ & $80.3 \pm 8.9$ & $80.2 \pm 7.6$ & 0.9427 \\
\hline Male, n (\%) & $94(45.9)$ & $50(49.0)$ & $44(42.7)$ & 0.3653 \\
\hline Grip strength & $18.5 \pm 9.0$ & $18.2 \pm 8.6$ & $18.8 \pm 9.4$ & 0.6649 \\
\hline Timed Up and Go & $14.6 \pm 12.0$ & $13.3 \pm 6.8$ & $15.8 \pm 15.4$ & 0.1521 \\
\hline Gait speed $(\mathrm{m} / \mathrm{sec})$ & $0.70 \pm 0.36$ & $0.70 \pm 0.38$ & $0.70 \pm 0.35$ & 0.9238 \\
\hline ERA score & $16.6 \pm 6.2$ & $16.7 \pm 6.5$ & $16.5 \pm 6.0$ & 0.8444 \\
\hline Kokmen mental status score & $34.5 \pm 2.3$ & $34.5 \pm 2.2$ & $34.4 \pm 2.4$ & 0.8550 \\
\hline Barthel ADL & $94.4 \pm 9.2$ & $94.3 \pm 9.7$ & $94.6 \pm 8.7$ & 0.8161 \\
\hline PHQ 9 score for depression & $3.7 \pm 3.8$ & $4.0 \pm 3.8$ & $3.4 \pm 3.7$ & 0.2463 \\
\hline SF-I 2 physical & $35.1 \pm 11.0$ & $35.5 \pm 10.7$ & $34.7 \pm 11.3$ & 0.5853 \\
\hline SF- 12 mental & $55.9 \pm 8.0$ & $54.8 \pm 8.7$ & $57.1 \pm 7.1$ & 0.0345 \\
\hline
\end{tabular}

Notes: Continuous variables reported as mean \pm standard deviation. Copyright $(\subset) 2012$, American Medical Association. All rights reserved. Adapted with permission from Takahashi PY, Pecina JL, Upatising B, et al. A randomized controlled trial of telemonitoring in older adults with multiple health issues to prevent hospitalizations and emergency department visits. Arch Intern Med. 2012;172(I0):773-779. ${ }^{6}$

Abbreviations: ERA, Elder Risk Assessment; ADL, activities of daily living; PHQ, Physical Health Questionnaire.

The time-to-event curves for both entry into hospice after study enrollment (Figure 2) and mortality (Figure 3) were not different between the two groups. Of the nine hospice patients in the telemonitoring group, seven were referred to local hospices and two were referred to the Mayo Hospice. In usual care, two of the four patients were referred to the Mayo Hospice. For patients in the Mayo Hospice, there was no difference in the number of hospice visits (face-to-face) between the intervention group (13.8 notes; $\mathrm{SD} \pm 24.4)$ and the usual care group (14.5 notes; $\mathrm{SD} \pm 17.4)$. Information on the number of visits to the regional hospices was not available.

The power analysis was calculated based on the above findings from this exploratory study. Using the outcome of hospice enrollment, one finds a $36 \%$ power to detect a difference in hospice enrollment between telemonitoring and usual care. This assumes a one-sided Chi-squared test of 0.05 with 9/94 patients in telemonitoring and 4/100 usual care patients. To attain $80 \%$ statistical power in the present study in order to detect a difference between both groups, the study would require 284 patients in each $\operatorname{arm}$ (total $\mathrm{n}=568$ ).

\section{Discussion}

In this analysis we explored at-risk older adults using telemonitoring versus those receiving usual care as part of their health care plan. We did not find any differences in the utilization of hospice care between patients who used telemonitoring and those who did not. Although this difference was not significant, more patients enrolled in hospice care overall, with 9 of 94 participants $(9.6 \%)$ in the telemonitoring group enrolling in a hospice, compared to 4 of 100 subjects $(4.0 \%)$ enrolling in a hospice in the usual care group $(P=0.12)$. There were no significant differences found between the groups regarding time until entry into hospice. The average and median times in hospice were longer in the usual care group compared to the telemonitoring group, but these results were also not significant. These findings are not conclusive and serve as initial pilot information for future work with community-based telemonitoring. To our knowledge, hospice enrollment has not been assessed as an outcome in previous telemonitoring studies. It should be noted that this pilot study was underpowered with a power calculation of $36 \%$ pertaining to the number of outcomes in

Table 2 Hospice referrals in telemonitoring and usual care in 205 older adults

\begin{tabular}{|c|c|c|c|c|}
\hline & $\begin{array}{l}\text { Overall } \\
n=194\end{array}$ & $\begin{array}{l}\text { Intervention } \\
\mathrm{n}=94\end{array}$ & $\begin{array}{l}\text { Usual care } \\
n=100\end{array}$ & P-value \\
\hline Number (\%) of patients in hospice during trial & $13(6.3)$ & $9(8.8)$ & $4(3.9)$ & 0.1207 \\
\hline Mean number of hospice visits during trial (SD) & $\mid 4.0(2 \mid .8)$ & $13.8(24.4)$ & | 4.5 ( 17.4$)$ & 0.9588 \\
\hline Median (IQR) & $2(0-23)$ & $2.0(0.0-25.0)$ & II $.5(0.0-32.0)$ & \\
\hline Mean time (days) to hospice entry during trial (SD) & $206.2(112.8)$ & $206.4(89.9)$ & $205.5(171.2)$ & 0.9923 \\
\hline Median (IQR) & $204(|3|-3||)$ & $204.0(148.5-305.5)$ & $224.5(38.0-354.0)$ & \\
\hline Mean days in hospice during trial (SD) & $76.8(106.1)$ & $57.9(99.2)$ & I $19.3(123.8)$ & 0.3580 \\
\hline Median (IQR) & $27(8-6 I)$ & $27.0(5.5-57.5)$ & $106.0(11.0-240.75)$ & \\
\hline
\end{tabular}

Abbreviation: IQR, interquartile range. 


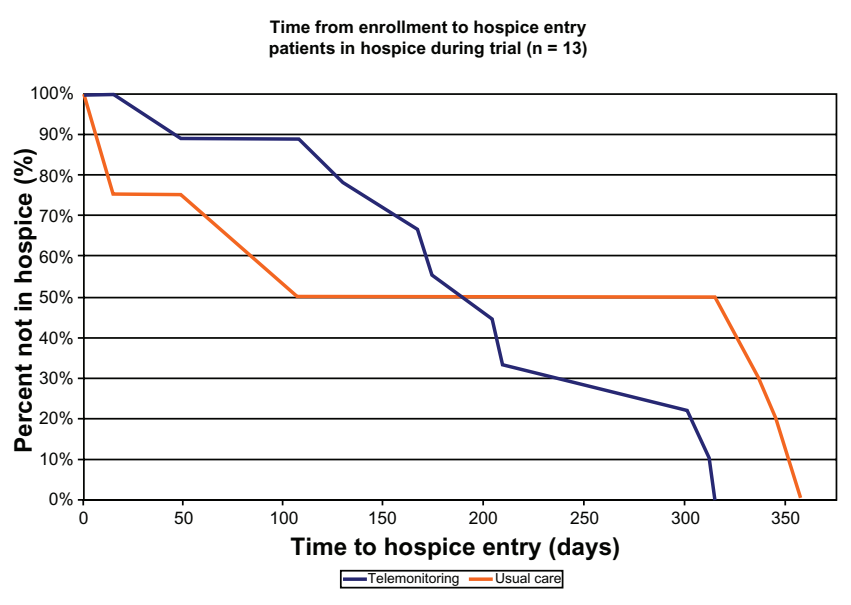

Figure 2 Time from enrollment to hospice entry among patients enrolled in hospice $(n=13)$.

our population. It is possible that different case management programs involving telemonitoring can help with end-oflife discussions and appropriate referrals to hospice. Given that high mortality rates are expected among older patients with complex, chronic health conditions, it is possible that hospice care might be appropriate for many individuals in this population.

The primary limitation of this study concerned the small number of subjects involved with this trial. Hospice enrollment was an uncommon event despite the number of medically complex patients. It should be noted that this analysis was a secondary evaluation of data from a previous randomized controlled trial, and it was not powered to detect a full effect. A second potential limitation is that this study did not employ a specific intervention through which to evaluate and enroll patients into hospice care. From this standpoint, a specific intervention or set of guidelines may have increased hospice enrollment. There are also other potential biases in

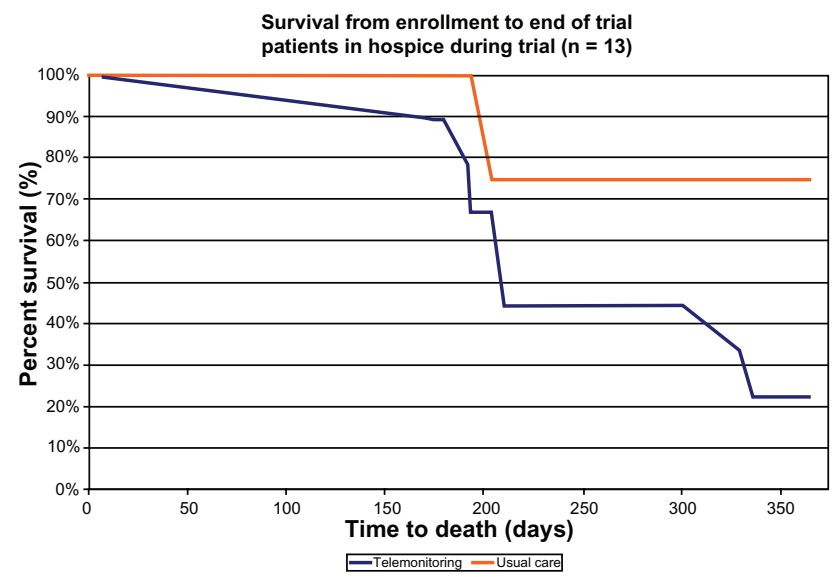

Figure 3 Survival from enrollment in hospice care $(n=13)$. this study. Subjects were not blinded because the presence of a telemonitoring device in the home prevented blinding. This could lead to the Hawthorne effect and to clinical differences in care due to more intensive monitoring. ${ }^{16}$ The psychological bias associated with telemonitoring could potentially shift the subjects towards health care that is more medically aggressive than hospice care. The primary care team was aware of the intervention (ie, the use of the telemonitoring device); however, the decision for hospice care was determined by clinical necessity. The entry into hospice was well documented in the EMR, and deaths that occurred during the study were also recorded. The Mayo Clinic has an extensive EMR with an informatics infrastructure, which captures outcomes like palliative care, hospice enrollment, and death. ${ }^{17}$ Lastly, measuring the number of home hospice visits performed by nurses as a secondary outcome was challenging because many patients were in a private hospice, and therefore we did not have access to the number of hospice visits received by the patients.

Although the evidence for hospice entry and telemonitoring are limited, previous studies have explored the relationship between case management and hospice enrollment. Heart failure is a common illness for case management and was the primary condition for the largest randomized trial in telemedicine. ${ }^{1}$ In patients dying of heart failure and who were eligible to be enrolled in a hospice, only $18.4 \%$ received hospice care. ${ }^{18}$ Within the nursing home population, a structured evaluation for goals of care resulted in $20 \%$ enrollment among the hospice evaluation intervention versus $1 \%$ in the usual care group. ${ }^{19}$ Some qualitative studies indicate that multiple barriers (like psychosocial factors) can affect hospice referral outside of medical eligibility. ${ }^{20}$ Despite these barriers, some studies have indicated that increased hospice referral arises from case management. A 2009 study of a comprehensive case management program resulted in increased numbers of patients accessing hospice care, and the average length of hospital stay also increased. The authors found that hospice referrals increased from $30.8 \%$ to $71.7 \%$ among group members who were in a case management program compared to controls, respectively. ${ }^{21}$ Thus, it may be possible that telemonitoring increases hospice referrals; however, future research will likely require a specific intervention to further demonstrate this effect, as the use of monitoring by itself may not be adequate.

One important finding from this study involves the overall length of stay (LOS) within hospice care. The overall average LOS in the cohort as a whole was 76.8 days (SD \pm 106.1 ), and within the telemonitoring cohort, the LOS was 57.9 days 
(SD \pm 99.2 ); these findings were compared to data from 2007 that reported an overall national cohort level LOS of 65 days. ${ }^{22}$ Thus, the study findings are consistent with national benchmark standards for average LOS and median LOS. Interestingly, the median LOS was 106 days in the usual care group, which was much longer than benchmark averages. Given the small number of overall hospice referrals $(n=4)$, it is difficult to draw conclusions from this finding. Many recognize that there appears to be a dichotomous pattern of short stays in hospice (just before death) and long stays in hospice..$^{23}$ In our study, we found a similar pattern, with the median and mean LOS days diverging considerably. One goal of telemonitoring would be to increase the utilization of palliative care and hospice services. We did not see this increase within our study, perhaps because the study was small and underpowered.

Future directions of this pilot study offer researchers the chance to answer a number of different questions. A larger trial with adequate numbers (potentially up to 568) will shed further light on the relationship between telemonitoring and hospice enrollment. Future studies should implement specific guidelines and infrastructure to increase hospice enrollment when appropriate. Furthermore, investigating palliative care and hospice outcomes in this high-risk group might be important for future work. More importantly, initiating a discussion surrounding palliative care and end-of-life issues might be appropriate for this population.

\section{Conclusion}

In this small, randomized controlled trial, patients who were randomized to telemonitoring did not enter hospice more frequently or spend a greater length of time in hospice. The study was small, with 205 subjects, and was underpowered. In spite of this, more people did enroll in hospice care after using telemonitoring services $(9.6 \%)$ compared to usual care $(4.0 \%)$. It is hoped that with larger trials, one can fully determine the relationship between telemonitoring and hospice enrollment.

\section{Acknowledgments}

We acknowledge Betty Wirt and Jenny Hurt for their help in study coordination. The authors would also like to thank Jody Clikeman from Mayo Clinic Grant and Publication Support Services for providing editorial assistance.

\section{Disclosure}

Intel provided the Intel ${ }^{\circledR}$ Health Guides and technical support. The authors received research support from the Mayo Clinic. No competing financial interests exist.

\section{References}

1. Chaudhry SI, Mattera JA, Curtis JP, et al. Telemonitoring in patients with heart failure. $N$ Engl J Med. 2010;363(24):2301-2309.

2. Woodend AK, Sherrard H, Fraser M, Stuewe L, Cheung T, Struthers C. Telehome monitoring in patients with cardiac disease who are at high risk of readmission. Heart Lung. 2008;37(1):36-45.

3. Trief PM, Teresi JA, Eimicke JP, Shea S, Weinstock RS. Improvement in diabetes self-efficacy and glycaemic control using telemedicine in a sample of older, ethnically diverse individuals who have diabetes: the IDEATel project. Age Ageing. 2009;38(2):219-225.

4. Takahashi PY, Tung EE, Crane SJ, Chaudhry R, Cha S, Hanson GJ. Use of the elderly risk assessment (ERA) index to predict 2-year mortality and nursing home placement among community dwelling older adults. Arch Gerontol Geriatr. 2012;54(1):34-38.

5. Demiris G, Oliver DP, Wittenberg-Lyles E, Washington K. Use of videophones to deliver a cognitive-behavioural therapy to hospice caregivers. J Telemed Telecare. 2011;17(3):142-145.

6. Takahashi PY, Pecina JL, Upatising B, et al. A randomized controlled trial of telemonitoring in older adults with multiple health issues to prevent hospitalizations and emergency department visits. Arch Intern Med. 2012;172(10):773-779.

7. Takahashi PY, Hanson GJ, Pecina JL, et al. A randomized controlled trial of telemonitoring in older adults with multiple chronic conditions: the Tele-ERA study. BMC Health Serv Res. 2010;10:255.

8. Crane SJ, Tung EE, Hanson GJ, Cha S, Chaudhry R, Takahashi PY. Use of an electronic administrative database to identify older community dwelling adults at high-risk for hospitalization or emergency department visits: the elders risk assessment index. BMC Health Serv Res. 2010;10:338.

9. Kokmen E, Naessens JM, Offord KP. A short test of mental status: description and preliminary results. Mayo Clin Proc. 1987;62(4):281-288.

10. Ware J Jr, Kosinski M, Keller SD. A 12-Item Short-Form Health Survey: construction of scales and preliminary tests of reliability and validity. Med Care. 1996;34(3):220-233.

11. Wade DT, Collin C. The Barthel ADL Index: a standard measure of physical disability? Int Disabil Stud. 1988;10(2):64-67.

12. Wang CY, Chen LY. Grip strength in older adults: test-retest reliability and cutoff for subjective weakness of using the hands in heavy tasks. Arch Phys Med Rehabil. Nov 2010;91(11):1747-1751.

13. Cesari M, Kritchevsky SB, Penninx BW, et al. Prognostic value of usual gait speed in well-functioning older people - results from the Health, Aging and Body Composition Study. J Am Geriatr Soc. 2005;53(10): 1675-1680.

14. Beauchet O, Fantino B, Allali G, Muir SW, Montero-Odasso M, Annweiler C. Timed Up and Go test and risk of falls in older adults: a systematic review. J Nutr Health Aging. 2011;15(10):933-938.

15. Podsiadlo D, Richardson S. The timed "Up and Go": a test of basic functional mobility for frail elderly persons. $J$ Am Geriatr Soc. 1991;39(2):142-148.

16. McCarney R, Warner J, Iliffe S, van Haselen R, Griffin M, Fisher P. The Hawthorne Effect: a randomised, controlled trial. BMC Med Res Methodol. 2007;7:30.

17. Herasevich V, Pickering BW, Dong Y, Peters SG, Gajic O. Informatics infrastructure for syndrome surveillance, decision support, reporting, and modeling of critical illness. Mayo Clin Proc. 2010;85(3):247-254.

18. Berry JI. Hospice and heart disease: missed opportunities. J Pain Palliat Care Pharmacother. 2010;24(1):23-26.

19. Casarett D, Karlawish J, Morales K, Crowley R, Mirsch T, Asch DA. Improving the use of hospice services in nursing homes: a randomized controlled trial. JAMA. 2005;294(2):211-217.

20. Walshe C, Chew-Graham C, Todd C, Caress A. What influences referrals within community palliative care services? A qualitative case study. Soc Sci Med. 2008;67(1):137-146.

21. Spettell CM, Rawlins WS, Krakauer R, et al. A comprehensive case management program to improve palliative care. J Palliat Med. 2009;12(9):827-832. 
22. Caffrey C, Sengupta M, Moss A, Harris-Kojetin L, Valverde R. Home health care and discharged hospice care patients: United States, 2000 and 2007. Natl Health Stat Report. 2011;38:1-27.
23. Connor SR. US hospice benefits. J Pain Symptom Manage. 2009;38(1): 105-109.

\section{Publish your work in this journal}

Clinical Interventions in Aging is an international, peer-reviewed journal focusing on evidence-based reports on the value or lack thereof of treatments intended to prevent or delay the onset of maladaptive correlates of aging in human beings. This journal is indexed on PubMed Central, MedLine, the American Chemical Society's 'Chemical Abstracts Ser-

\section{Dovepress}

vice' (CAS), Scopus and the Elsevier Bibliographic databases. The manuscript management system is completely online and includes a very quick and fair peer-review system, which is all easy to use. Visit $\mathrm{http}: / / \mathrm{www}$.dovepress.com/testimonials.php to read real quotes from published authors.

Submit your manuscript here: http://www.dovepress.com/clinical-interventions-in-aging-journal 